\title{
Endovascular Treatment
}

\section{of a Distal Aneurysm of the Superior Cerebellar Artery by Intra-Aneurysmal Injection of Glue}

\author{
M. LEONARDI, L. SIMONETTI, A. ANDREOLI* \\ Servizio di Neuroradiologia, U.O. Neurochirurgia*, Ospedale Bellaria; Bologna
}

Key words: brain aneurysm, glue embolisation, endovascular treatment

\section{Summary}

Aneurysms in the distal cerebellar arteries are rare events. They are associated with a poor prognosis, as surgery or embolisation with Guglielmi detachable coils (GDCs) is very difficult. The ability to treat them surgically depends on the location of the aneurysm, but surgery is considered difficult and is associated with a high morbidity/mortality rate.

Embolisation with GDCs may be difficult or impossible because of the distal location of the aneurysm or the unfavourable ratio between the size of the aneurysm and the size of the parent vessel.

We report our experience in one case treated with intra-aneurysmal injection of glue.

The aneurysm, located in the distal right superior cerebellar artery, was catheterized with a flow-guided microcatheter, and glue was slowly injected into the aneurysmal sac.

The treatment resulted in total occlusion of the aneurysm with preservation of the parent artery.

\section{Introduction}

Aneurysms of the distal superior cerebellar artery (SCA) are rare events. Locksley et $\mathrm{al}^{7}$, in a series of 2349 intracranial aneurysms, found six aneurysms of the SCA $(0.3 \%)$. Gacs et $\mathrm{al}^{5}$, in a surgical series of 910 vertebrobasilar aneurysms, reported six distal aneurysms of the SCA $(0.7 \%)$.

The ability to treat them surgically depends on the location of the aneurysm, but surgery is considered difficult and is associated with a rather high morbidity/mortality rate ${ }^{1,10}$. Embolization with Guglielmi detachable coils (GDCs, Target Therapeutics, Fremont, CA) may be difficult or impossible:

- first, it can be difficult to catheterize the aneurysm with the microcatheters we normally use for this purpose, the Excel 14 or the Tracker 10 (Target Therapeutics), owing to the very distal location of the aneurysm;

- second, because of the large width of the aneurysmal neck and the small diameter of the parent artery, occlusion of the aneurysm with GDCs would most likely result in a thrombosis of the parent vessel, due to its catheterization or protrusion of the coils;

- third, this kind of aneurysm often shows a very irregular shape that is not likely to adapt itself to the coil bulging.

We report our experience in one case of distal right SCA aneurysm treated with intraaneurysmal injection of glue, with the technique previously described (in 1999) by Cognard et $\mathrm{al}^{3}$ in three patients. 


\section{Case Report}

A 44-year-old woman sought medical attention for frequent headaches. CT examination disclosed a "suspect right perimesencephalic aneurysmatic malformation". Findings at neurologic examination were normal.

Cerebral angiography confirmed a distal right SCA aneurysm (figure. 1A,2A,3A); angiography revealed a significant contrast stagnation (figure 1B). The shape was quite irregular, with a "pseudomycotic" aspect. Initially, endovascular treatment with GDCs was considered, but we anticipated several problems with that technique because of the distal location of the aneurysm and the unfavorable ratio between the size of the aneurysm and the size of the parent vessel and its irregular shape made packing of the aneurysm using coils difficult and unsafe.

Consequently, we decided to perform an endovascular treatment with injection of glue.

A $6 \mathrm{~F}$ guiding catheter was introduced, with its tip placed in the left vertebral artery. Through the guiding catheter, the distal superior cerebellar artery was catheterized with a flow-guided Flowrider 1.8 microcatheter (Micro Therapeutics, Irvine, CA) directed by a Silver Speed 0.010F microguidewire (Micro Therapeutics). The tip of the microcatheter was gently introduced into the aneurysm. A mixture of $1 \mathrm{ml}$ Histoacryl (Braun, Melsungen, Germany) and $2 \mathrm{ml}$ of iodized oil (Lipiodol, Andre Guerbert, Aulnay sous Bois, France) was prepared in a $3 \mathrm{ml}$ Luer-Lock syringe and very slowly injected into the aneurysm. Control angiography (figure 1C,D, 2B,C, 3B,C) showed occlusion of the aneurysm with preservation of the parent artery and normal flow in the distal hemispheric branch. A small remnant of the aneurysm was seen at the level of the neck. After awakening from anesthesia, the patient was asymptomatic and was discharged five days later. Follow-up angiography at three months showed complete occlusion of the aneurysm with no visible neck remnant. The patient is scheduled for a threeyear follow-up angiogram to confirm the stability of the outcome.

\section{Discussion and Conclusion}

Aneurysms located in the distal intracranial vessels are rare. Distal aneurysms represent approximately $7 \%$ to $9 \%$ of anterior cerebral artery aneurysms and $2 \%$ to $7 \%$ of middle cerebral artery aneurysms. Regarding the distal segments of the cerebellar artery, Locksley et $\mathrm{al}^{7}$, in a series of 2349 intracranial aneurysms, found six aneurysms of the SCA $(0.3 \%)$ and 11 of the PICA $(0.5 \%)$. Gacs et $\mathrm{al}^{5}$, in a surgical series of 910 vertebrobasilar aneurysms, reported six distal aneurysms of the SCA $(0.7 \%)$ and eight aneurysms of the PICA $(0.9 \%)$.

The poor prognosis in patients with ruptured intracranial aneurysms of the posterior circulation was documented in 1995 by Schievink et $\mathrm{al}^{8}$. In their series, the 48 -hour survival rate was $32 \%$ for aneurysms of the posterior circulation and $77 \%$ for aneurysms of the anterior circulation.

Outcome after surgery, depending on the location of the aneurysm, has been reported by several investigators. Gacs et $\mathrm{al}^{5}$ reported 16 patients with distal cerebellar aneurysms, all of whom were in good clinical condition when they underwent surgery. The final outcome was considered excellent or good in 11 cases $(69 \%)$ and poor in four cases $(25 \%)$.

Since the introduction of GDCs, it is generally believed that ruptured or unruptured aneurysms in the posterior circulation may be treated with GDCs, with a lower morbidity / mortality risk than surgery. The main limitation to this treatment is the morphology of the aneurysm, the presence of a wide neck being the most important limitation.

In our case of distal SCA aneurysms, we judged GDC treatment to be unsuitable for the reasons previously described. We decided to perform the endovascular treatment with the technique described by Cognard et $\mathrm{al}^{3}$. This technique consists in intra-aneurysmal injection of glue, after catheterization using a flowguided microcatheter, with the over-the-wire approach.

Several authors have reported the results of experimentally occluded aneurysms using cyanoacrylate, either by direct puncture of the aneurysm or by an endovascular approach ${ }^{6,9}$. The major drawback reported in these studies has been the large number of distal embolizations of cyanoacrylate into normal vessels.

Fox et $\mathrm{al}^{4}$ reported the results from three patients with cerebral aneurysms who were treated with isobutyl 2-cyanoacrylate combined with calibrated-leak balloons. All treatments resulted in major infarction and death. Szikora 


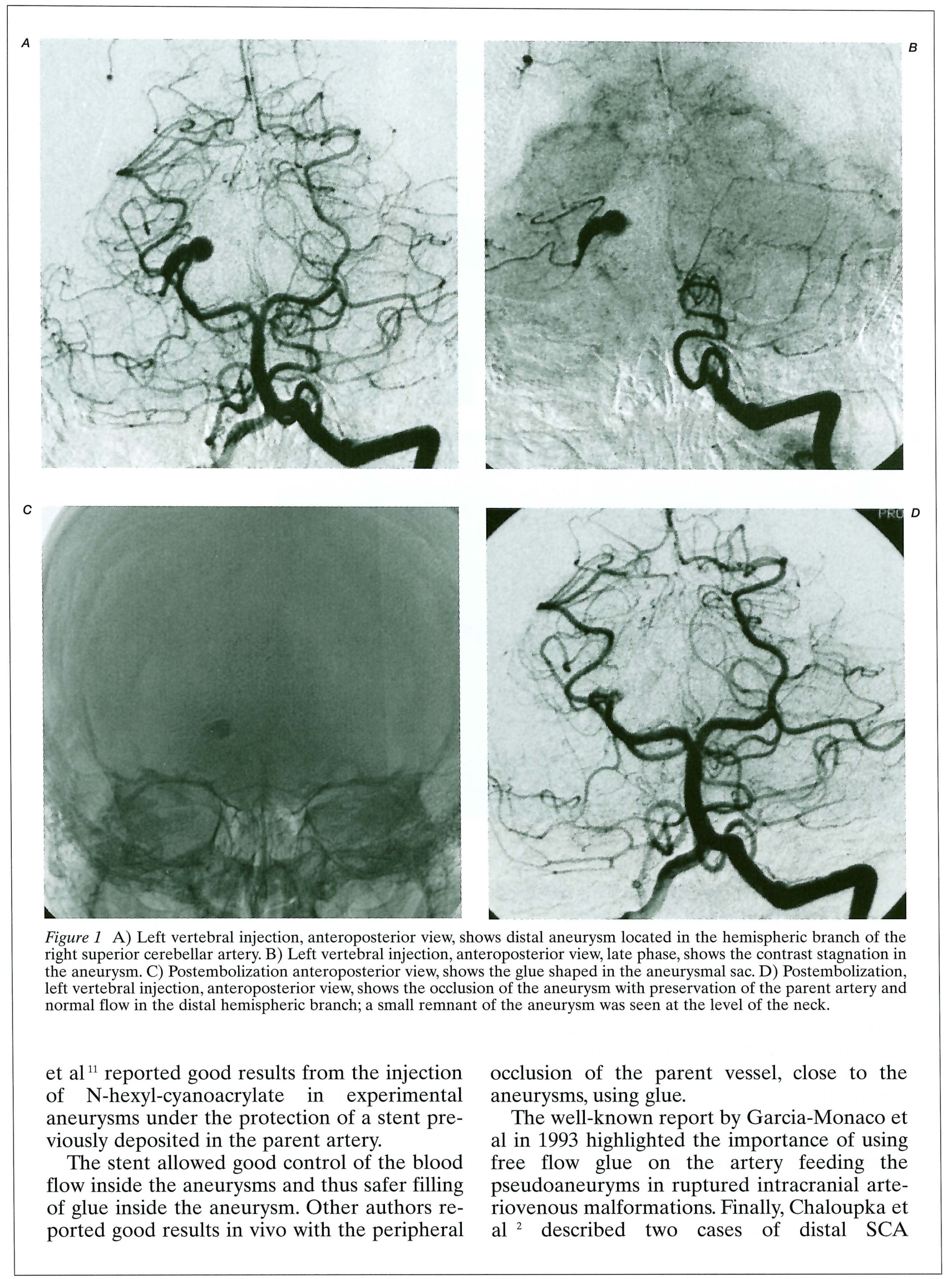



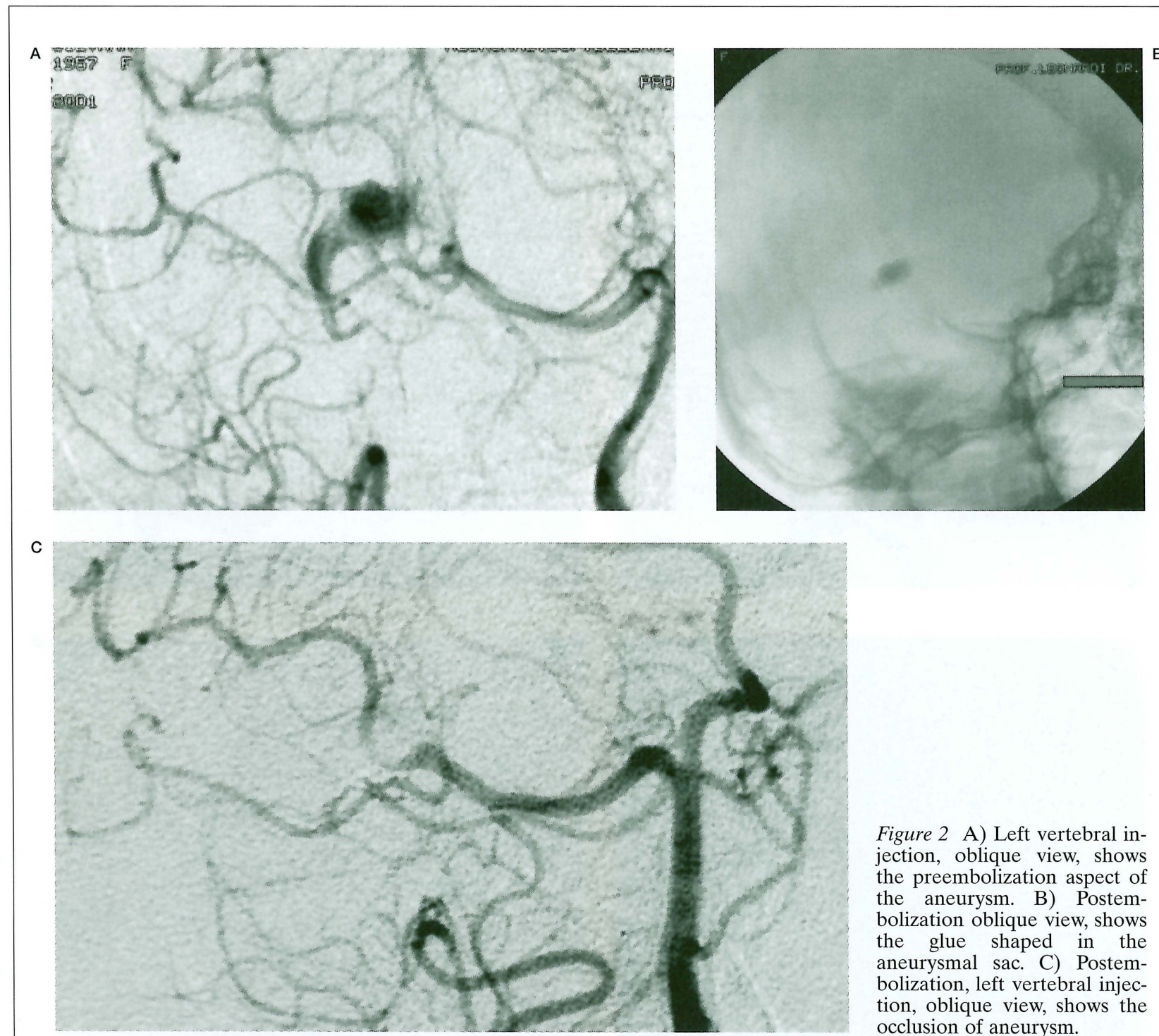

Figure 2 A) Left vertebral injection, oblique view, shows the preembolization aspect of the aneurysm. B) Postembolization oblique view, shows the glue shaped in the aneurysmal sac. C) Postembolization, left vertebral injection, oblique view, shows the occlusion of aneurysm.

aneurysms, treated successfully with distal occlusion of the parent vessel using glue.

In accordance with Cognard ${ }^{3}$, some aspects of the technique merit attention.

Microcatheterization. The technique allows easy and safe catheterization of an aneurysm using a flow-guided microcatheter, because of the flexible and atraumatic tip. It was performed with the use of a Flow Rider flow-guided microcatheter directed by a $0.010 \mathrm{~F}$ Silver Speed microguidewire. The tip of the microcatheter, shaped with steam, was placed inside the aneurysm and kept there. The microcatheter was slowly pushed over the wire, with the tip never coming into contact with the aneurysma1 wall. With this technique, catheterization of the aneurysmal sac is completely atraumatic. In our case, the microcatheterization was quite easy and safe.

The aim of embolization. It was initially to occlude the aneurysm and the parent artery in front of the aneurysmal neck. Otherwise, the proximal portion of the SCA, as well as the perforating arteries arising from these arterial segments, would remain patent and the distal segments of the SCA would be retrogradely vascularized from the very rich pial collateral circulation. The slow injection of contrast material inside the aneurysm allowed us to obtain 


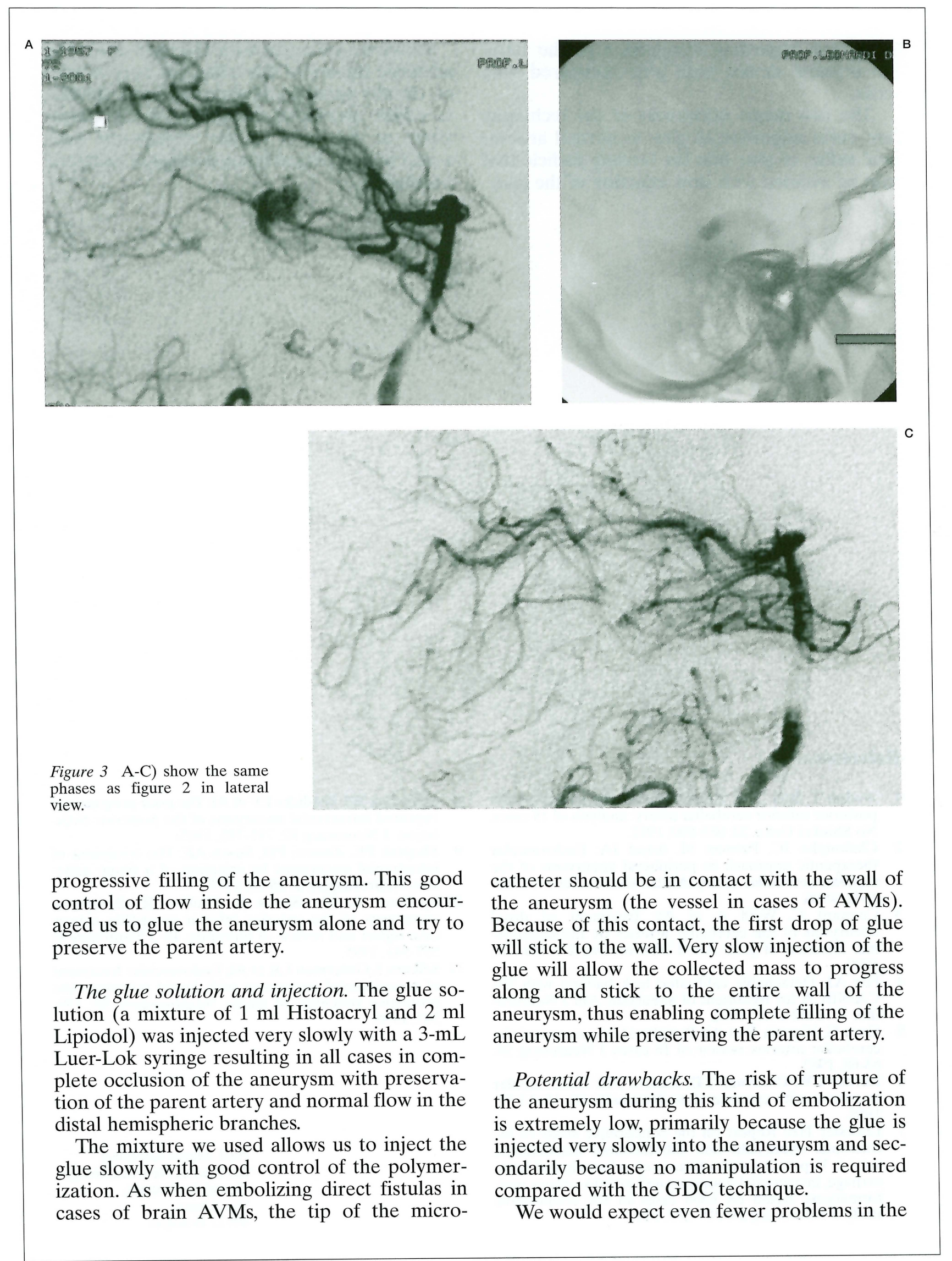


long-term regarding compaction of the intraaneurysmal device with glue compared with coils.

The two major drawbacks of the technique are distal migration of glue to normal arteries and reflux of glue into the feeding pedicle that can be avoided with slow injection of the glue.
However, we agree with Cognard et al. who advocate the use of this technique only in cases of distally located aneurysms in small arteries, in which accidental occlusion of the parent artery at the level of the aneurysmal neck would not be critical because of good collateral circulation.

\section{References}

1 Andoh $\mathrm{T}$, Itoh $\mathrm{T}$ et $\mathrm{Al}$ : Peripheral aneurysms of the posterior inferior cerebellar artery: analysis of 15 cases. No Shinkei Geka 20: 683-690, 1992.

2 Chaloupka JC, Putman M, Awad IA: Endovascular therapeutic approach to peripheral aneurysms of the superior cerebellar artery. Am J Neuroradiol 17: 1338$1342,1996$.

3 Cognard C, Weill A et Al: Treatment of distal aneurysms of the cerebellar arteries by intraaneurysmal injection of glue. Am J Neuroradiol 20: 780-784, 1999.

4 Fox AJ: Detachable calibrated leak balloon with IBCA/NBCA for treatment of aneurysm. Am J Neuroradiol 13: 1452-1453, 1992

5 Gacs G, Vinuela F et Al: Peripheral aneurysms of the cerebellar arteries: review of 16 cases. J Neurosurg 58: 63-68, 1983.

6 Kerber CW, Cromwell LD, Zanetti PH: Endovascular treatment with cyanoacrylate. Neurosurgery 16: 13-17, 1985.

7 Locksley HB: Report on the cooperative study of intracranial aneurysms and subarachnoid hemorrhage, section V, part I: natural history of subarachnoid hemorrhage, intracranial anurysms and arterio-venous malformations; based on 6368 cases in the cooperative study. J Neurosurg 25: 219-239, 1966.
8 Schievink WI, Wijdicks EF et Al: The poor prognosis of ruptured intracranial aneurysms of the posterior circulatjon. J Neurosurg 82: 791-795, 1995.

9 Sheptak PE, Zanetti PH, Susen AE: The treatment of intracranial aneurysms by injection of a tissue adhesive. Neurosurgery 1: 25-29, 1977.

10 Spallone A, De Santis S, Giuffre R: Peripheral aneurysms of the anterior inferior cerebellar artery: case report and review of literature, Br J Neurosurg 9: 537-541, 1995.

11 Szikora I, Guterman LR et Al: Endovascular treatment of experimental aneurysms with liquid polymers: the protective potential of stents. Neurosurgery 38: 339347, 1996.

Prof. Marco Leonard Servizio di Neuroradiologia Ospedale Bellaria,

Via Altura

40122 Bologna 